\title{
Education 4.0: An remote approach for training of intelligent automation and robotic during COVID19
}

\author{
H. Smajic 1[https://orcid.org/0000-0002-0669-1979], T. Duspara 1[https://orcid.org/0000-0002-6985-5950] \\ ${ }^{1}$ Technische Hochschule Köln
}

\begin{abstract}
The COVID-19 pandemic confronts universities with great challenges to maintain research and teaching activities with as little contact as possible. Lecturers currently have to migrate to Internet teaching. In most cases, e-learning and digital tools are used to continue online courses to replace classroom teaching. But current online approaches are limited to just lectures and theoretical mathematical exercises. In this paper it will be shown how practical exercises can be carried out remotely via internet in a real technical environment. Experimental laboratory equipment for automation technology and mechatronics is always associated with high costs. The reason for the high investments are the costs for different intelligent devices within an automation solution and the costs for extensive engineering. Beyond the costs, the number of workstations usually does not correspond to the required number of students to be trained. In this case, the same exercises have to be repeated several times, which also leads to in-creased personnel costs.

Remote laboratories are a very cost-effective solution for these problems. This paper describes how this goal can be achieved by implementing a WBT server (WBT - Web-Based Training Server) and a Java-based client-server architecture. The idea behind a remote controlled laboratory is to use web technologies and the Internet as communication infrastructure to perform an experimental part of the training with programmable automation devices. First of all, a detailed requirement profile for the laboratory was developed. Primarily technical, didactical and organizational requirements are concerned. In addition, the laboratory is to improve the education of the students by interactive, problem-oriented learning on real industrial automation components. The aim of the training is to learn suitable working methods for the design (engineering) of complete automation solutions starting from simple to medium complex machines and plants.
\end{abstract}

Keywords: Education 4.0, Remote Lab, IloT, COVID-19

\section{Introduction}

The use of online automation engineering in general has thus far not found any sweeping acceptance on the operating side. The engineering capable of being performed via worldwide data paths and by means of multi-media tools are thus far advances of innovative manufacturers of capital goods who, in this way, ensure themselves new unique selling points compared with their competitors.

This type of manufacturer-driven approach neglects important customer interests through the proprietary design and the differing manners of communication of the systems. The reasons for this trend lie in the fact that many manufacturers have initially striven for support by their own service personnel, especially during the commissioning phase. The finding phase of the suppliers with respect to online engineering that is still continuing makes the path towards a uniform communications standard for use in the industrial environment more difficult. The latter has been prevented in particular through the rapid changes in the field of various information and communication technologies [1]. 
Some concepts for the provision of diverse machine-related services via remote control of these machines by humans with information technology tools have already been developed. These constitute comprehensive solutions for IT-assisted service scenarios with the involvement of the organizational structures of manufacturer and operating company that above all make clear the complete host of services that can be reproduced on the basis of modern-day information and communication technologies [2]. The use of the communications infrastructure of the Internet itself is becoming increasingly established in this field and, as a result, will develop far-reaching potential for the future online engineering.

Nevertheless, the introduction of online engineering brings with it major challenges for the companies. Many of these companies are placing their faith in differing suppliers in the field of machines and machine control, with the result that the large number of heterogeneous components and online service solutions offered, prevents any efficient organization of remote access that represents a further precondition for a comprehensive, inter-compatible and future-oriented introduction of online service in the field of remote control access. Operating companies of Online supportable, production systems are consequently faced with high infrastructure and organizational costs as they constantly have to administer the large number of different systems in order to keep them available for possible use.

In this respect, the world-wide operating, major manufacturers of online engineering systems offer comprehensive services that have already been taken into consideration in the planning of the installations, however, the well-known problems of the proprietary solutions and the difficulty with integration into company infrastructures continue to exist. In detail the following weaknesses of current remote. In detail the following weaknesses can be shown:

- $\quad$ Users are confronted with different online service solutions.

- Insufficient compatibility to allow in-house usage of proprietary systems.

- High requirements for safety of operator and machine.

- $\quad$ High requirements for security of system access and network.

- Version conflicts due to different software updates.

- $\quad$ Current complexity allows operation by experts only.

\section{Available automation concepts}

In process and manufacturing automation, the demands for increasing productivity and flexibility while maintaining consistently high quality standards are constantly growing in the face of intensifying competition. This development leads to increasingly complex requirements for control concepts and implies a further increase in the complexity of automation tasks. In order to meet these continuously changing requirements, decentralized control concepts are subject to constant change towards distributed systems with open interfaces. This change is also favored significantly by the daily growing performance potential of microelectronics and information technology [3, 4]. As a result, numerous centralized and decentralized control concepts have emerged in automation technology, which can be classified primarily according to their topological, functional and communication aspects (Figure 1). 


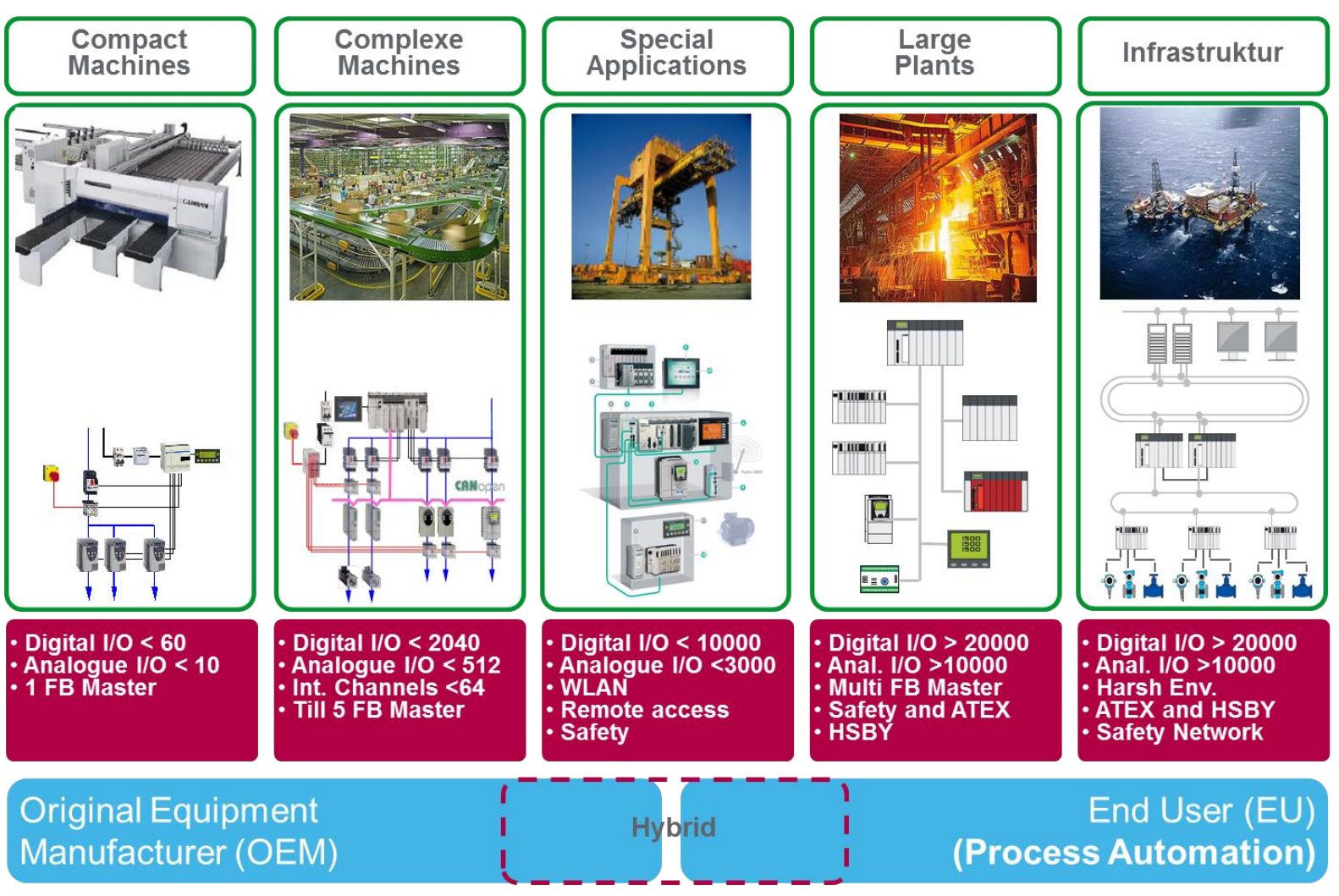

Figure 1. Available automation concepts for machine and plants

For the control tasks of simple machines, which predominantly require binary signal processing in the form of logic or sequence control, central programmable logic controllers are still used. In complex machines and plants, a decentralized and modular network of individual controllers is increasingly replacing centralized structures. These are usually small and medium-sized control systems or intelligent sensors and drives directly on the machine or plant, which are networked with each other via appropriate field buses. In order to protect employees and the environment, safety-oriented control components and safety-oriented communication systems are used in complex plants and in the field of infrastructure applications. A continuous process is guaranteed by the use of redundant and highly available systems (HSBY).

A new generation of decentralized control systems is significantly influenced by the spread of industrial Ethernet and web technologies. By consistently integrating these technologies, various problems of existing fieldbuses, such as openness and manufacturer independence, can be solved. These protocols have been successfully introduced right down to the control level. It is undisputed that this trend towards further integration will continue right into the field level. The introduction of Ethernet and the shifting of control functionalities to the field devices and drives requires further functional changes to the controllers and control systems when they are integrated into the higher automation structures, which consequently also affects the engineering and thus their software tools. The specification of different device descriptions (FDT, GDS, EDS, etc.), which are made available to the user by corresponding user organizations, is particularly helpful for the integration of these technologies.

The design of an automation solution depends of machine size and market segment, where automated equipment can be applied. The main criteria for building of automation architecture are: number of digital and analogue Inputs/Outputs, intelligent channels, type of fieldbus, remote access, high availability and safety. 


\section{Training in real and virtual environment}

The currently most widespread model for knowledge transfer at European universities is still characterized by passive lectures and exercises. However, such knowledge transfer through theoretical input in engineering courses always suffers from a low recall rate. At the Faculty of Vehicle Systems and Production, application-oriented teaching is provided with the support of practical exercises and group work. Although the recall rate with this approach is as high as $32 \%$ after three months, the number of dropouts is still too high. One of the main reasons for this dropout rate is an excessively high level of abstraction in the transfer of knowledge in mechatronic modules. This problem makes it increasingly difficult to find candidates who can carry out internal project work in the form of individual projects, interdisciplinary projects and master projects. The current shortage of skilled workers in Germany is also intensify-ing the competition between universities and industry, since even the few existing candidates with specialist knowledge prefer to write their theses in industry.

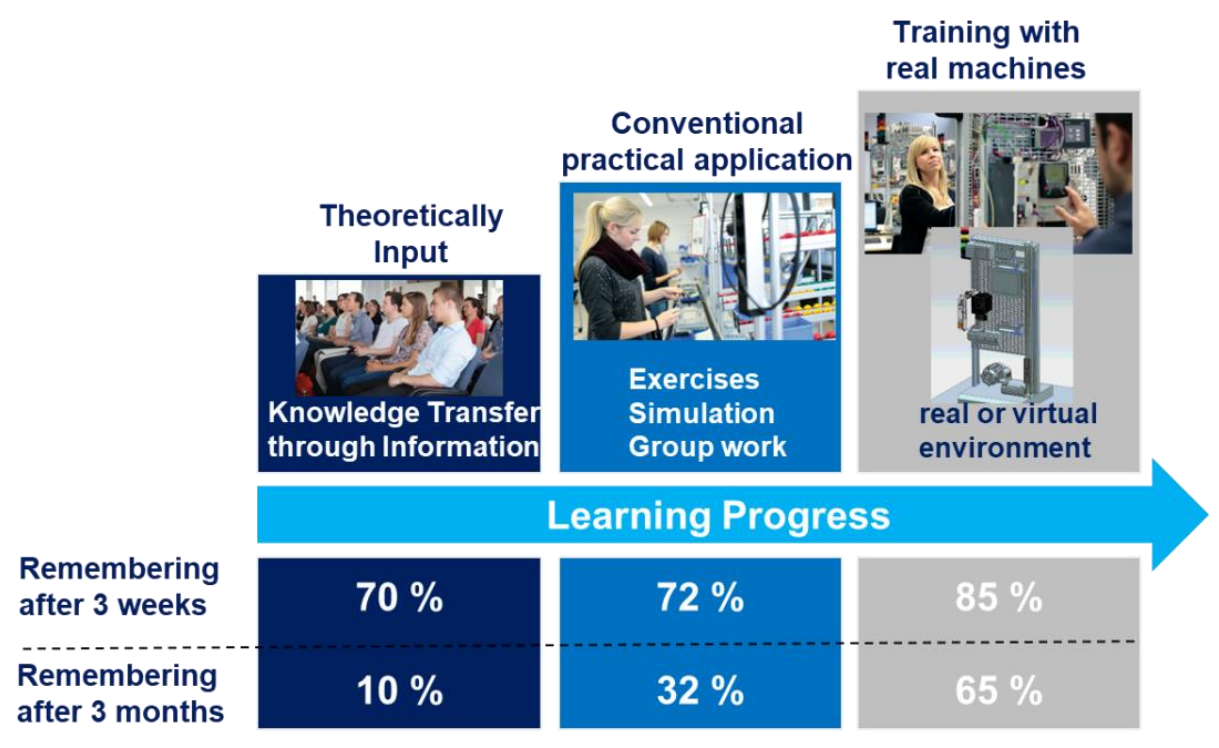

Figure 2. Various training opportunities in higher education

The main focus of this project is the development of teaching content with a high practical relevance for areas of automation technology. The previous rather passive and theoretical learning should be supplemented by the experience in the practical environment and would lead to essentially better and more efficient learning results.

For this purpose, technical workstations have been developed which enable active experience and experimentation. Such an approach can make the level of abstraction of complex programming tasks much more comprehensible through personal experience with all sensory organs and through cooperation and communication with others. It can even increase the above-mentioned recall rate to $65 \%$ and thus lead to significantly better learning outcomes.

\section{Design of an automation solution for practical education}

With the aim of providing students with a modern education in the field of automation technology, the Cologne University of Applied Sciences and Schneider Electric GmbH have designed a range of equipment to meet the needs of the students. Care was taken to ensure that as many and innovative automation technologies as possible were taken into account. Because of the investment security, the selected components should not be on the market 
for longer than two years. A classification of these technologies, which had to be considered, is shown in Figure 3.
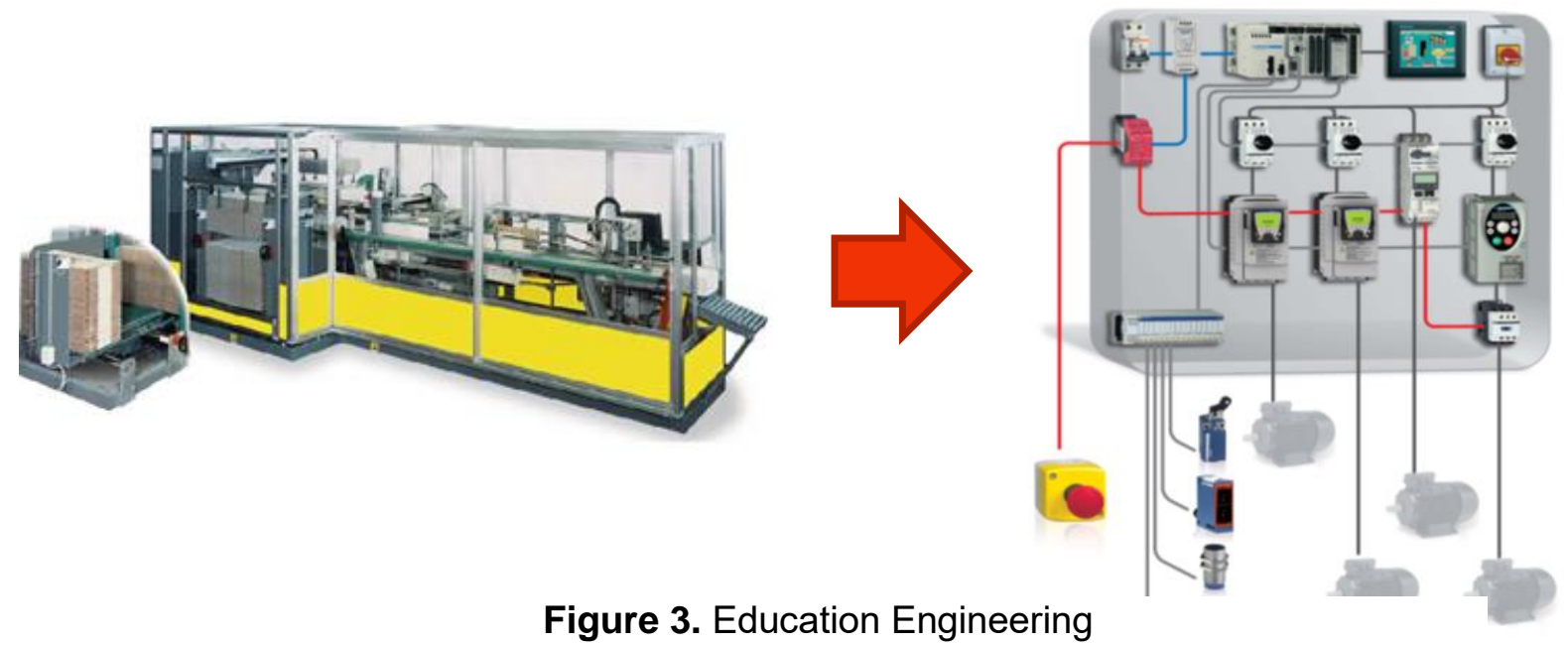

Figure 3. Education Engineering

Inductive and capacitive sensors, optical encoders and data carriers for radio frequency identification (RFID) are provided for the acquisition of binary and analogue process signals and are connected to the programmable logic controller (PLC). A modern mid-range PLC with numerous external modules was selected for processing the process signals. For the tasks "operating and monitoring", sophisticated HMI touch panels with colour display are used. Each station is equipped with modern electronic drive components. A frequency converter is used to practice clockwise and counter-clockwise rotation and commands for constant speeds and constant torque. A linear axis with servo motor and servo controller can be used to perform various tasks with positioning control. All components are networked via CANOpen fieldbus. Profibus DP is also available as an option. In this way, students can configure field buses and learn about their communication protocols. All stations are connected to each other via Industrial Ethernet, so that access to web servers of individual components and technologies of web-based automation can be explained.

The Cologne University of Applied Sciences is one of the first universities in Germany that would like to sensitize its students to the efficient use of electrical energy as part of their university education. For this purpose, appropriate measuring devices were installed at each station, with which all energy data of three consumers are continuously recorded and stored. Subsequently, these data are to be evaluated and analysed by the students. After the analysis, proposals for measures to be introduced to save energy will be developed. For training of automation engineering skills we designed a suitable working stations, which consider those commonly technologies. One workstation contains following equipment:

- $\quad$ Sensors, detectors, encoders and RFID switches for data detection

- $\quad$ Programmable logic control (PLC) for data processing

- Human Machine Interface

- Velocity speed driver and Motion driver

- Smart meter for energy efficiency

- $\quad$ Ethernet Network and CANOpen as machine bus

- $\quad$ Programming and SCADA software 
In order to increase the motivation of the students for the education in the field of automation technology even more, technologies were also procured with which young people can identify more easily today. This makes it possible for students to complete certain parts of the exercises from their own smartphones using Java Apps. An overview of this is shown in Figure 3. For example following exercises can be performed:

- $\quad$ Command for motor via Bluetooth

$>$ Left turn and Right turn

$>$ Change velocity

- $\quad$ Commands vie GSM Modem

$>$ Email sending from process

$>$ Alarm per SMS receive and email

In addition to the hardware, appropriate site licenses were provided for the complete engineering (programming, visualization with SCADA, simulation, etc.). The entire apparatus equipment of the Laboratory for Automation Technology at the Cologne University of Applied Sciences comprises 20 modern workstations of identical design. A large number of practical exercises can be carried out in the teaching environment. The training of students in the areas of control, drive and communication technology is thus decisively improved and made much more clearly understandable.

\section{IloT architecture for remote education}

The concept presented above was built identically at 20 workstations in the laboratory. At each station, two students work in a team, so the capacity for training is 40 participants. In order to use the potential of the equipment also outside, the approach of loT was implemented. At each workstation 10 global IP addresses were implemented. A WBT server manages an Ethernet network of more than 200 addresses. The Internet-based architecture allows remote access to the automation technology modules for exercises and practical training. The resources of the laboratory can be used as "Distance Learning" for locationindependent training. This technology offers an enormous advantage, especially in the global pandemic period due to COVID-19. Students do not have to be present at the university, but are able to carry out their automation tasks (programming, visualization, parameterization etc.) online via re-mote access. The equipment is also available to our external partners from industry and other universities.

In the middle of the remote architecture is a server that manages the entire information content of the individual stations. The server controls remote access and routing to individual workstations as well as authentication (user name and password) of the users. Various SQL databases for archiving access protocols are implemented on the server. Remote access from the global network is established via a VPN connection. In this step the external computer becomes part of the laboratory network and is assigned an IP address via the DHCP server. 


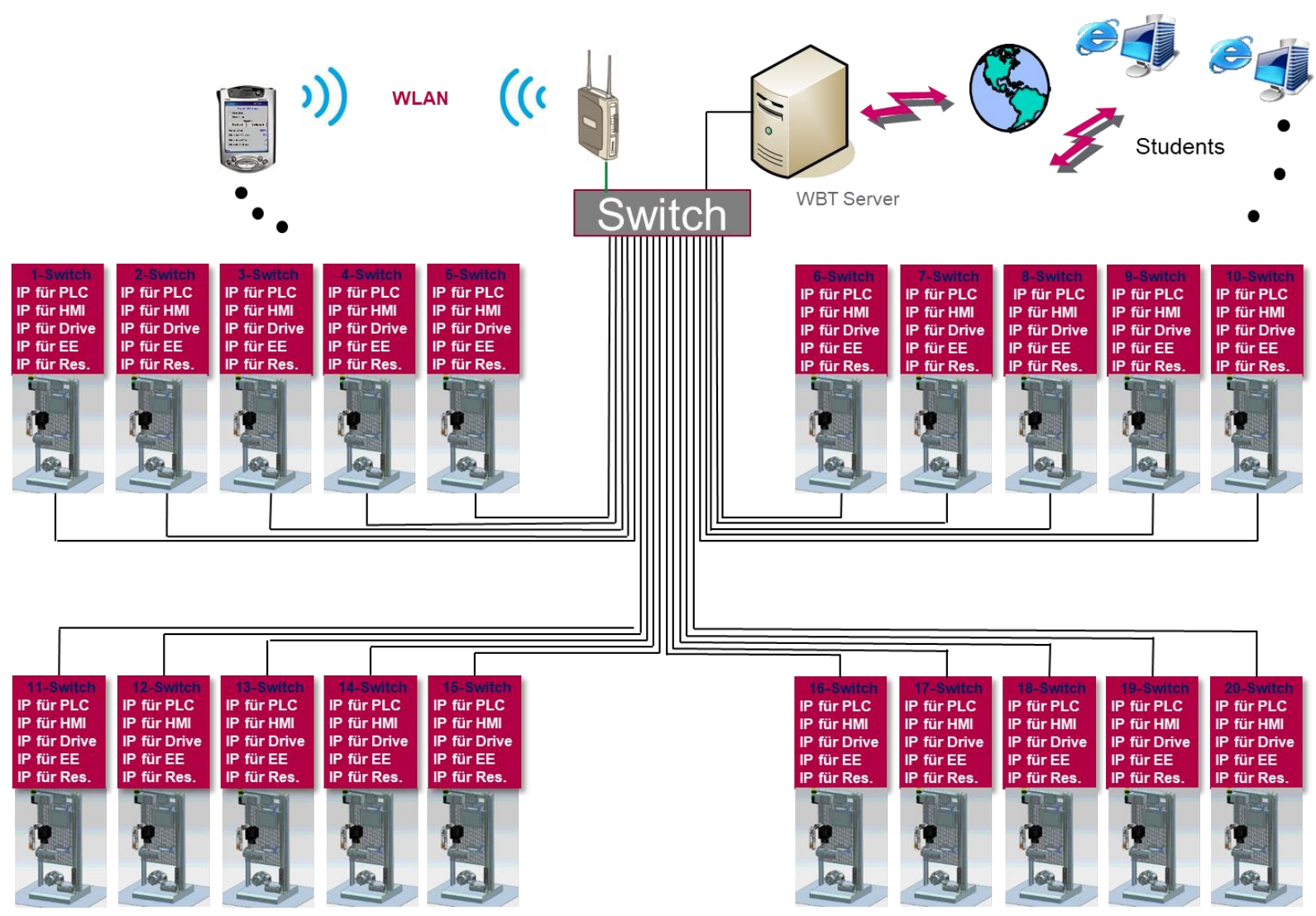

Figure 4. loT architecture for practical exercises

In the next step, a connection to a virtual computer from the computer pool is created by establishing a remote desktop connection according to previously defined rules. This computer provides a working environment with all software components required for programming. After a "user name and password" query, access is gained to all programming software for PLC, HMI and drives. The user can observe the downloading and testing of the programs written on the automation components via a standard webcam.

Once a student has logged on to a remote computer, he can use all of Toll's soft-ware to program the platforms. In figure 4 on the left side is the software for configuring and programming the PLC. Five different languages (LD, FBD, SFC, IL, ST) according to IEC 61131-3 are available for programming the tasks. When the tasks have been programmed, the participant can test and validate his solutions in a de-bugging process before the project is remotely transferred to the hardware. Tools for diagnostics and online services are then also available. Following a similar pattern, students also use the software to create the screens on the HMI device. Objects are defined in a graphic editor and linked to control variables.

HMI software uses Ethernet TCP/IP connectivity and is therefore able to support decentralized WEB gate access as well as the exchange of application data between terminals, the transfer of recipes and protocols for variables and much more.

For the developed application, a simulation of the PLC variables ( $/ / O$, internal bits and words) and the graphical application can be performed with a RunTimer, before downloading the application to the device. This creates a condition window (top right) where all the exercise programs are validated. A webcam (bottom left) shows all activities on the unit. 


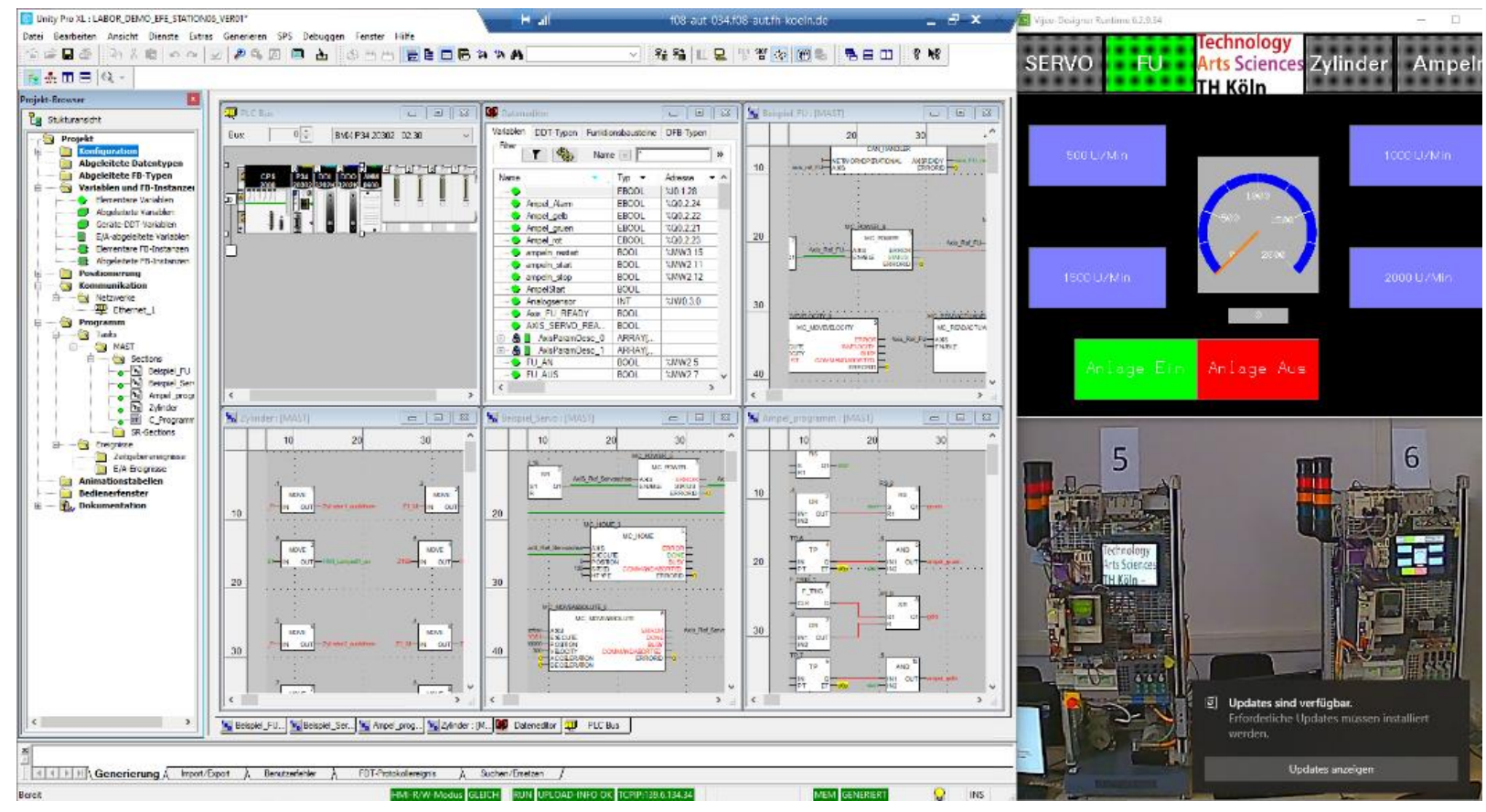

Figure 5. Software tools, testing and validation of programs remotly

\section{Conclusion and future challenge}

The first experiences have shown that the interest and motivation of students for the tasks of automation engineering has increased significantly. Varied exercises reduce the degree of abstraction in programming tasks significantly and lead to a significant improvement of the examination results.

The developed internet-based architecture with its WEB server provides a good basis for location-independent access to all resources of the laboratory. The challenges of digitization in teaching is another focus of current research at the faculty. Re-search projects include, for example, the development of innovative assistance systems for evaluating learning progress based on artificial intelligence approaches and the introduction of an automatized booking service (Scheduler Tools). The resources of the laboratory are currently also being used by partners from industry and partners from universities as "Distance Learning".

\section{References}

[1] Restivo M, Mendes J, Lopes A, Silva C, Chouzal F. A Remote Laboratory in Engineering Measurement. IEEE Transactions on Industrial Electronics. 2009 Dec;56(12):4836-4843. https://doi.org/10.1109/tie.2008.2011479

[2] Niedersteiner S, Lang J, Pohlt C, Schlegl T. Klassifikation des Arbeitsfortschritts. atp magazin. 2017 Nov 17;59(11):58-66. https://doi.org/10.17560/atp.v59i11.1911

[3] Osinde NO, Byiringiro JB, Gichane MM, Smajic H. Process Modelling of Geothermal Drilling System Using Digital Twin for Real-Time Monitoring and Control. Designs. 201908 17;3(3):45. https://doi.org/10.3390/designs3030045

[4] Smajic H, Bosco J. SIMULATION TOOLS FOR VIRTUAL PLC-TESTING IN EXTRUSION PROCESS. University of Banja Luka. DEMI 2019; Bosnia and Hercegovina. 2019 May.

[5] Falkman P, Helander E, Andersson M. Automatic generation: A way of ensuring PLC and HMI standards. ETFA2011. Factory Automation (ETFA 2011). 201109.

https://doi.org/10.1109/etfa.2011.6059201 